\title{
A utilização da síntese verde para obtenção de nanopartículas de prata a partir de extratos de girassol (helianthus annuus)
}

The use of green synthesis for obitenting silver nanoparticles from sunflower extracts (helianthus annuus)

El uso de síntesis verde para obtener nanopartículas de plata a partir de extractos de girasol (helianthus annuus)

Matheus Henrique da Silva Lemos ORCID: https://orcid.org/0000-0002-3554-0141 Universidade Federal do Piaú, Brasil E-mail: lemosmhs@gmail.com

Girlene Soares de Figueirêdo

ORCID: https://orcid.org/0000-0002-6710-1549 Universidade Federal do Piauí, Brasil

E-mail: girlenesf@gmail.com

Ravena da Silva Portela

ORCID: https://orcid.org/0000-0002-8582-752X Centro Universitário UniFacid, Brasil

E-mail: ravenaportela2012@hotmail.com

Ana Flávia Machado de Carvalho ORCID: https://orcid.org/0000-0002-6691-4808 Centro Universitário UniFacid, Brasil

E-mail: anaflaviaparaibana@ hotmail.com

\begin{abstract}
Resumo
As nanopartículas de prata (AgNPs) têm chamado grande atenção em pesquisas recentes, devido a sua variedade de aplicação em diversas áreas. Essas nanopartículas podem ser produzidas por diferentes métodos utilizando a síntese verde (green synthesis). O que resulta na minimização do custo de produção das nanopartículas. Dessa forma, este trabalho teve como objetivo sintetizar nanopartículas de prata (AgNPs) estabilizada em extratos de girassol (Helianthus annus). A produção da nanopartícula foi realizada em agitação sobre uma chapa magnética aquecida até a configuração total da nanoparticula, ao final foi utilizado o extrato vejetal para a estabilização da mesma. As mesmas foram caracterizadas através do UV-VIS. A analise antimicrobiana das nanoparticulas foi feita em placa de petri com a medição do tamanho do halo inibitório formado. Obteve-se AgNPs a dimensão nos espectros de UV-vis em torno de 20 nanometros. Verificou-se através dos picos de absorção que variou - $414 \mathrm{~nm}$ a $422 \mathrm{~nm}(\lambda \mathrm{max} 422$ $\mathrm{nm})$ que o extrato de Helianthus annuus foi capaz de estabilizar com efeito de formação de halo sobre as linhagens de $S$. aureus $(16 \mathrm{~mm}), E$. coli $(12 \mathrm{~mm})$ e P. aeruginosa $(10 \mathrm{~mm})$, enquanto as AgNPs que não foram estabilizadas com o girassol não apresentaram o halo inibitório. Através desta pesquisa foi possível comprovar que a síntese verde (SV), apesar ser um novo produto nanotecnológico com bases sustentáveis é uma excelente alternativa com uma alta reprodutibilidade e está de acordo com os princípios da química verde, apresentando-se como uma alternativa de baixo custo, eco-amigável e biocompatível na síntese de AgNPs. Além de servir como subsidio para pesquisas posteriores.
\end{abstract}

Palavras-chave: Síntese verde; Nanopartículas de prata; Nanotecnologia. 


\begin{abstract}
Silver nanoparticles (AgNPs) have attracted great attention in recent research, due to their variety of application in several areas. These nanoparticles can be produced by different methods using green synthesis. What results in the minimization of the production cost of the nanoparticles. Thus, this work aimed to synthesize silver nanoparticles (AgNPs) stabilized in sunflower extracts (Helianthus annus). The production of the nanoparticle was carried out in agitation on a magnetic plate heated until the total configuration of the nanoparticle, at the end the vejetal extract was used to stabilize it. They were characterized using UV-VIS. The antimicrobial analysis of the nanoparticles was performed in a petri dish with the measurement of the size of the inhibitory halo formed. AgNPs were obtained in the UV-vis spectra around 20 nanometers. It was verified through the absorption peaks that it varied - $414 \mathrm{~nm}$ to 422 $\mathrm{nm}(\lambda \max 422 \mathrm{~nm})$ that the extract of Helianthus annuus was able to stabilize with effect of formation of halo on the lines of S. aureus $(16 \mathrm{~mm})$, E coli $(12 \mathrm{~mm})$ and P. aeruginosa $(10 \mathrm{~mm})$, while AgNPs that were not stabilized with sunflower did not present the inhibitory halo. Through this research it was possible to prove that green synthesis (SV), despite being a new nanotechnological product with sustainable bases, is an excellent alternative with a high reproducibility and is in accordance with the principles of green chemistry, presenting itself as an alternative of low cost-effective, eco-friendly and biocompatible in the synthesis of AgNPs. In addition to serving as a subsidy for further research.
\end{abstract}

Keywords: Green synthesis; Silver nanoparticles; Nanotechnology.

\title{
Resumen
}

Las nanopartículas de plata (AgNP) han atraído gran atención en investigaciones recientes debido a su variedad de aplicaciones en varias áreas. Estas nanopartículas se pueden producir mediante diferentes métodos utilizando síntesis verde. Lo que se traduce en la minimización del coste de producción de las nanopartículas. Así, este trabajo tuvo como objetivo sintetizar nanopartículas de plata (AgNPs) estabilizadas en extractos de girasol (Helianthus annus). La producción de la nanopartícula se realizó en agitación sobre una placa magnética calentada hasta la configuración total de la nanopartícula, al final se utilizó el extracto vejetal para estabilizarla. Se caracterizaron mediante UV-VIS. El análisis antimicrobiano de las nanopartículas se realizó en una placa de Petri con la medida del tamaño del halo inhibidor formado. Los AgNP se obtuvieron en los espectros UV-vis alrededor de 20 nanómetros. Se verificó a través de los picos de absorción que varió - $414 \mathrm{~nm}$ a $422 \mathrm{~nm}$ ( $\lambda$ max $422 \mathrm{~nm})$ que el extracto de Helianthus annuus fue capaz de estabilizarse con efecto de formación de halo en las líneas de $\mathrm{S}$. aureus $(16 \mathrm{~mm}), \mathrm{E}$ coli $(12 \mathrm{~mm})$ y P. aeruginosa $(10 \mathrm{~mm})$, mientras que los AgNP que no se estabilizaron con girasol no presentaron halo inhibitorio. A través de esta investigación se pudo comprobar que la síntesis verde (SV), a pesar de ser un nuevo producto nanotecnológico con bases sostenibles, es una excelente alternativa con una alta reproducibilidad y está de acuerdo con los principios de la química verde, presentándose como una alternativa de bajo costo-efectivo, ecológico y biocompatible en la síntesis de AgNPs. Además de servir como subvención para futuras investigaciones.

Palabras clave: Síntesis verde; Nanopartículas de plata; Nanotecnologia.

\section{Introdução}

As nanopartículas são materiais que possuem suas dimensões na nanoescala entre 1-100 nanômetros, entretanto na biotecnologia e na medicina essa definição é mais flexível, dependendo da aplicação (Ratner, 2013).

Uma alternativa para a sua fabricação de nanopartículas é a síntese verde, que se mostra cada vez mais uma tecnologia em crescimento. As nanopartículas de prata podem ser produzidas em uma única etapa utilizando materiais oriundos de recursos renováveis, sem subprodutos indesejáveis e com ótimo custo-benefício (Souza, Souza \& Franchi, 2019).

A síntese de nanopartículas de prata vem despertando atenção por possuir uma gama de aplicações devido às suas propriedades e aplicações na área da saúde, tem se destacado entre pesquisas devido ao seu comportamento superparamagnético. Estes materiais podem ser empregados em várias aplicações que englobam desde materiais de armazenamento magnético, (mishra et al., 2016).

De acordo com Dasa et. al, (2019) A nanobiotecnologia verde é enriquecida na medida em que utiliza princípios da química verde para o desenvolvimento sustentável. No entanto, esta ciência está em sua infância e precisa se promover de modo a desenvolver tecnologias ecologicamente corretas e economicamente viáveis, que são as necessidades do momento na direção do desenvolvimento sustentável. Pode-se definir nanobiotecnologia verde quando baseada em princípios limpos de sínteses de nanopartículas, como, por exemplo, a partir de processos biotecnológicos. 
A Síntese verde, síntese biológica e síntese biogênica são termos comumente usados para a síntese ecologicamente correta de nanopartículas. Normalmente, as nanopartículas são sintetizadas através de métodos químicos, físicos e biológicos. A via de síntese biológica tem sido efetuada, sobretudo, através do uso de bactérias, fungos, plantas e cianobactérias. Técnicas a partir de extrato de plantas são simples de sintetizar nanopartículas (Amkhande, Ghule, Bamer, et al. 2019).

As plantas produzem biomoléculas funcionais, interessantemente, todas as partes das plantas podem ser usadas de modo eficiente para sintetizar nanopartículas, como as folhas, caules, sementes, frutos, látex entre outros. A biomassa morta e seca da planta também pode ser usada para a síntese bem sucedida de nanopartículas. Além disso, atuam como agentes protetores e estabilizadores das nanopartículas. O mecanismo exato da síntese de nanopartículas por extratos de plantas ainda não foi elucidado, observa-se resultados satisfatórios com o uso de aloe vera (babosa), Cinnamomum camphora (árvore de cânfora), curcumina, extrato de girassóis (Helianthus annuus) entre outros são alguns exemplos de síntese de nanopartículas de prata utilizando extratos de folhas (Ratner, et. al, 2013).

Deshmukha, et. al, (2019) sugere que a biossíntese utilizando extrato de plantas é mais adequado para produção em grande escala do que a utilização de micro-organismos, segundo o autor as plantas são mais seguras e levam menos tempo para realizar a síntese, já os micro-organismos muitos são patogênicos e levam vários dias para conseguirem reduzir as nanoparticulas. Embora haja crescente investimento em pesquisa para o uso da síntese verde, a escassez de estudos relacionados ao tema limita as fontes do presente estudo. Tornando-se assim promissor e de grande relevância científica.

Neste sentido, a pesquisa teve como objetivo geral sintetizar nanopartículas de prata (AgNPs) estabilizada em extratos de girassol (Helianthus annuиs).

\section{Metodologia}

\subsection{Procedimentos éticos}

A pesquisa não precisou ser submetida ao CEUA (Comitê de Ética em Uso de Animais) da UNIFACID, nem ao CEP (Comitê de Ética em Pesquisa), antes de iniciar a coleta de dados e desenvolvimento da pesquisa, por não se tratar de uma pesquisa com seres humanos nem animais. Respeitando assim os procedimentos éticos exigidos no trabalho.

\subsubsection{Cenário do estudo e coleta dos dados}

O trabalho foi desenvolvido no laboratório da UniFacid em parceria com o laboratório de química pertencente ao Instituto Federal do Piauí (IFPI). Realizada entre os meses de março e abril de 2020 inicialmente e finalizada no ano de 2021. Destaca-se que para coleta foi respeitada as recomendações da Organização Mundial de Saúde (OMS), relacionada a distanciamento social, referente à pandemia do Covid-19. As amostras de linhagens microbianas foram analisadas no mês de março de 2021, no laboratório de microbiologia da UFPI (Universidade Federal do Piauí), obedecendo aos protocolos de segurança estabelecidos.

\subsubsection{Método de pesquisa}

O método utilizado foi a síntese verde, que é chamado dessa forma exatamente por não gerar efeitos negativos ao meio ambiente. Trata-se de uma pesquisa Experimental de abordagem descritiva laboratorial. De acordo com Gil (2012), a pesquisa experimental constitui o delineamento mais prestigiado nos meios científicos, que determina um objeto de estudo selecionando variáveis capazes de definir formas de controle e observar efeitos que as variáveis relacionadas ao objeto de estudo, fazendo com que o pesquisador se torne um agente ativo e não um observador passivo para assim, atingir os resultados esperados. O pesquisador deve fazer uso de aparelhos e instrumentos com técnicas modernas para alcançar as variáveis de 
causa e efeito obtidas no objeto de estudo. O pesquisador precisa manipular pelo menos um dos fatores que se acredita ser responsável pela ocorrência do fenômeno que está sendo pesquisado.

Para a elaboração das nanopartículas de prata, alguns materiais foram utilizadas e estão descritos abaixo:

- Um envelope com 800mg de sementes de girassol Helianthus annuus- Isla LOTE 003000919.

- Uma solução de citrato de sódio 1\%, (pureza $\geq 99,0 \%$, lote MKBD2163V, ACS Regent, Sigma-Aldrich)

\subsection{Produção do extrato de sementes de girassol}

Para realização desta etapa de produção do extrato foi comprado um envelope com 800mg de sementes de girassol Helianthus annuиs- Isla LOTE 003000919, com época de semeadura de aproximadamente seis meses possuindo ciclo de 60 verões e com um numero aproximado de sementes de $20 \mathrm{~g}$.

Posteriormente foram pesados $10 \mathrm{~g}$ de sementes de girassol macerado e incorporados em $100 \mathrm{~mL}$ de água destilada e mantidas em repouso por 24 horas. Após esse período foi realizado uma rota-evaporação para a obtenção do extrato concentrado que foram distribuídos e envasado em frascos vidro âmbar para impedir a perda de qualquer componente fotossensível até o momento do seu uso na estabilização das nanopartículas de prata durante o processo da sua produção.

Figura 1 - Sequencia experimental que mostra a produção do extrato de sementes de girassol.
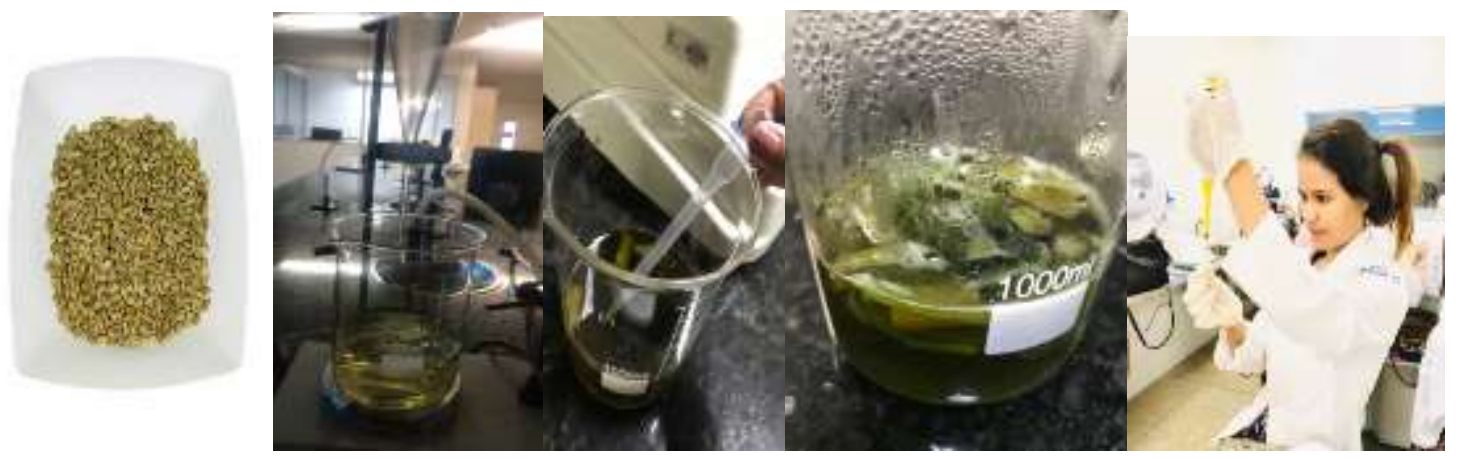

Fonte: Arquivo de Dispositivo Móvel (Autora)

$\mathrm{Na}$ sequência de fotos acima, que foi realizada entre os meses de março e abril de 2020, o laboratório de química pertencente ao Instituto Federal do Piauí (IFPI) em parceria com o laboratório da UNIFACID, podemos observar os grãos de extrato de girassol (Helianthus annuиs) que foram pesados, macerados e incorporados em $100 \mathrm{~mL}$ de água destilada e mantidas em repouso posteriormente, na forma de suspensão aquosa, apresentando assim baixo impacto ambiental.

\subsection{Síntese de nanopartículas de prata estabilizadas em Helianthus annuus (AgNP-Ha)}

Sobre agitação magnética numa chapa aquecedora, uma solução de $125 \mathrm{~mL}$ de $\mathrm{AgNO}_{3}(1,0 \mathrm{mmol} / \mathrm{L})$ foi aquecida até entrar em ebulição. Atingida a temperatura de ebulição, $5,0 \mathrm{~mL}$ de uma solução de citrato de sódio $1 \%$, (pureza $\geq 99,0 \%$, lote MKBD2163V, ACS Regent, Sigma-Aldrich) foi gotejada na solução de $\mathrm{AgNO}_{3}$. O gotejamento ocorreu numa taxa de 1,0 gota por segundo. A mistura mantida sobre aquecimento e agitação foi mudando de cor até atingir uma coloração amarelo acastanhado. Essa coloração indica a redução da prata de $\mathrm{Ag}^{+}$para $\mathrm{Ag}$ com formação das nanopartículas. Após atingir essa coloração, foi desligado o aquecimento da chapa aquecedora, no entanto, a solução foi mantida sobre agitação magnética. Após a solução contendo as nanopartículas atingir a temperara ambiente $\left(25^{\circ} \mathrm{C}\right)$ foi adicionado a mistura, o extrato das sementes de girassol previamente produzida. Após 15 minutos, a solução de $\mathrm{AgNO}_{3} /$ citrato/girassol foi armazenada num frasco âmbar. 
Deve-se ressaltar que nas reações realizadas houve variação na coloração o que é um bom indício da formação de nanopartículas de prata é a forte coloração amarelo/ambar na amostra, instantes após a etapa de adição do agente estabilizador, conforme podemos observar na Figura 2.

Figura 2- Sequência da formação química e aspecto visual da solução de nanopartícula de prata estabilizadas em Helianthus annuus (AgNP-Ha)
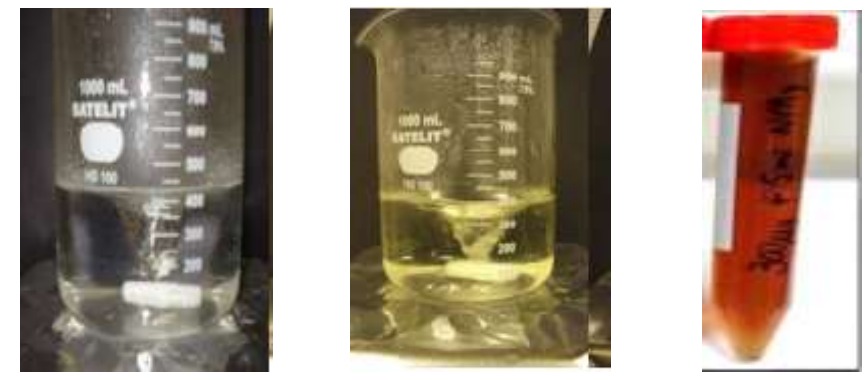

Fonte: Arquivo de Dispositivo Móvel (Autora)

A cor amarela característica observada na prata coloidal é resultado da absorção da radiação eletromagnética. Com a elevação das partículas de prata, a cor amarela característica da solução padrão passa para laranja e, posteriormente, alaranjada até atingir a coloração característica da prata em escala macroscópica (marron acastanhada). Esse fenômeno de diferença de coloração pode ser explicado pela adição de solução de nitrato de prata, que faz com que as partículas se aglomerem e se tornem maiores. (Junior et al., 2013).

As nanopartículas obtidas através da redução por extratos de plantas são na sua maioria esféricas, mas através de outros agentes químicos podem apresentar formatos variados como cubos, triângulos, bastões e até dendritos (Khodashenas et al., 2015).

\subsection{Caracterização e confirmação das nanopartículas}

A confirmação da síntese da nanopartícula foi realizada pele método de espectroscopia de luz ultravioleta visível (UV-Vis), os espectros foram obtidos em um espectrofotômetro modelo UV-6100S da Allcrom com feixe duplo. Os parâmetros de analises serão: faixa de varredura de 1900-900nm, abertura de fenda $=2,0 \mathrm{~nm}$ e resolução espectral de $1,0 \mathrm{~nm}$. Foram utilizados cubetas de quartzo com caminho óptico de $1,0 \mathrm{~cm}$ e as amostras foram dispersas em água com o auxílio de banho ultrassônico.

\subsection{Linhagens microbianas}

Os testes de verificação de atividade antimicrobiana da AgNP-Ha foram realizados contra cepas microbianas padrão. Sendo uma espécie bacteriana gram-positiva, Staphylococcus aureus ATCC 25923, uma espécie gram-negativa, Escherichia coli ATCC 25922, e uma espécie de fungo leveduriforme, Candida albicans ATCC 10231. Além das cepas padrão, também se determinou a atividade antimicrobina do referido composto contra uma cepa de Pseudomonas aeruginosa ATCC 27853, isolada de água de tratamento hospitalar.

As cepas bacterianas foram mantidas em tubos contendo ágar Brain Infusion Heart (BHI, Himedia, Índia) inclinados a $4{ }^{\circ} \mathrm{C}$, e antes do ensaio as células foram cultivadas durante uma noite a $37{ }^{\circ} \mathrm{C} \mathrm{em}$ Brain Heart Infusion (BHI, Himedia, Índia). O ágar Sabouraud Dextrose foi usado tanto para manutenção da cepa de levedura como para o cultivo das células. 


\subsection{Preparo do inóculo microbiano}

O inóculo das espécies microbianas que foram testadas foi padronizado pelo método de suspensão direta das colônias CLSI (2009) em solução salina esterilizada. Todas as culturas semeadas foram incubadas à temperatura de $37^{\circ} \mathrm{C}$ em estufa de crescimento. Por meio de uma alça de platina esterilizada realizou-se a inoculação dos micro-organismos repicados, em tubos de ensaio com $1 \mathrm{ml}$ de soro fisiológico a $0,9 \%$. A suspensão de células foi ajustada a concentração de $1 \times 10^{8} \mathrm{UFC} / \mathrm{mL}$, que corresponde a solução padrão de McFarland de 0,5.

\section{Resultados e Discussão}

\subsection{Síntese verde das nanopartículas de prata}

A formação de AgNPs por síntese verde utilizando o extrato de girassol (Helianthus annuus) foi realizada a partir da mistura da solução de $\mathrm{AgNO} 3$ na concentração final de $1 \mathrm{mM}$ com o EV na concentração de $10 \%$ (v/v), foi aquecida até entrar em ebulição. Atingida a temperatura de ebulição, $5,0 \mathrm{~mL}$ de uma solução de citrato de sódio $1 \%$, (pureza $\geq 99,0 \%$, lote MKBD2163V, ACS Regent, Sigma-Aldrich) foi gotejada na solução de $\mathrm{AgNO}_{3}$.

A análise espectroscópica de absorção na faixa UV-vis (ultravioleta-visível) consiste em um método simples e amplamente utilizado na determinação da formação de AgNPs e NPs-Ha em uma dispersão, devido à ressonância plasmônica de superfície dessas NPs (Chaudhuri e Paria, 2012). Essa máxima da absorção é dependente do diâmetro médio das NPs, possuindo intensidade maior quando as NPs possuem o raio em torno de $20 \mathrm{~nm}$ (Georgiev et al., 2013).

Geralmente o diâmetro das NPs obtidas por rotas de síntese verde utilizando extratos de plantas é descrito por meio de dados obtidos por MET (Nath e Banerjee, 2013). Quando 63 são realizadas medidas de DLS, geralmente não são mostrados os dados referentes ao extrato controle, provavelmente porque este também possui substâncias as quais também se organizam em nanoescala. No presente trabalho, optou-se pela metodologia de DLS para analisar as NPs em suspensão, por MFA para as análises quantitativas e MET para confirmar os dados obtidos pelas outras técnicas.

\subsection{Caracterização Visual}

Estudos sugerem que quando há presença de nanopartículas de prata na solução formada, a solução se torna mais escura, indo de transparente para um amarelo acastanhado. A sequência da Figura 1 mostra fotografias das amostras contendo nanopartículas de prata produzidas na presença do extrato de girassol concentrações de citrato de prata e com diferentes tempos de reação.

\subsection{Espectroscopia síntese e caracterização das nanopartículas}

A caracterização das AgNPs foi feita através de espectroscopia UV-VIS obtiveram resultados promissores e inéditos, uma vez que não há na literatura nenhum relato sobre a identificação de proteínas na sintase e/ou na estabilização em Helianthus annuиs para aplicações bactericidas. Na Figura 3 traz a faixa padrão de cada uma das substâncias utilizadas para a síntese e estabilização das nanopartículas, tanto o extrato de girassol quanto a solução de citrato de sódio $1 \%$ não apresentam qualquer processo de absorção de luz na região visível, enquanto a absorção de luz para a solução de nitrato de prata é muito pequena, podendo também ser desconsiderada e consequentemente ser citadas como soluções incolores.

Essa caracterização inicial da solução de produção como incolor se dá pelo princípio da qual as partículas de pratas são produzidas, pois nesse processo de redução para prata, em que ao ocorrer a transformação do nitrato de prata para as nanopartículas de prata ocorre uma mudança de coloração, em geral para um marrom escuro, que será detectada e quantificada de acordo com a quantidade produzida na síntese (Ravindra et al., 2010; Al-Shabib et al., 2020). 
Figura 3: Gráfico UV-Vis das substâncias usadas na produção e estabilização das AgNPs.

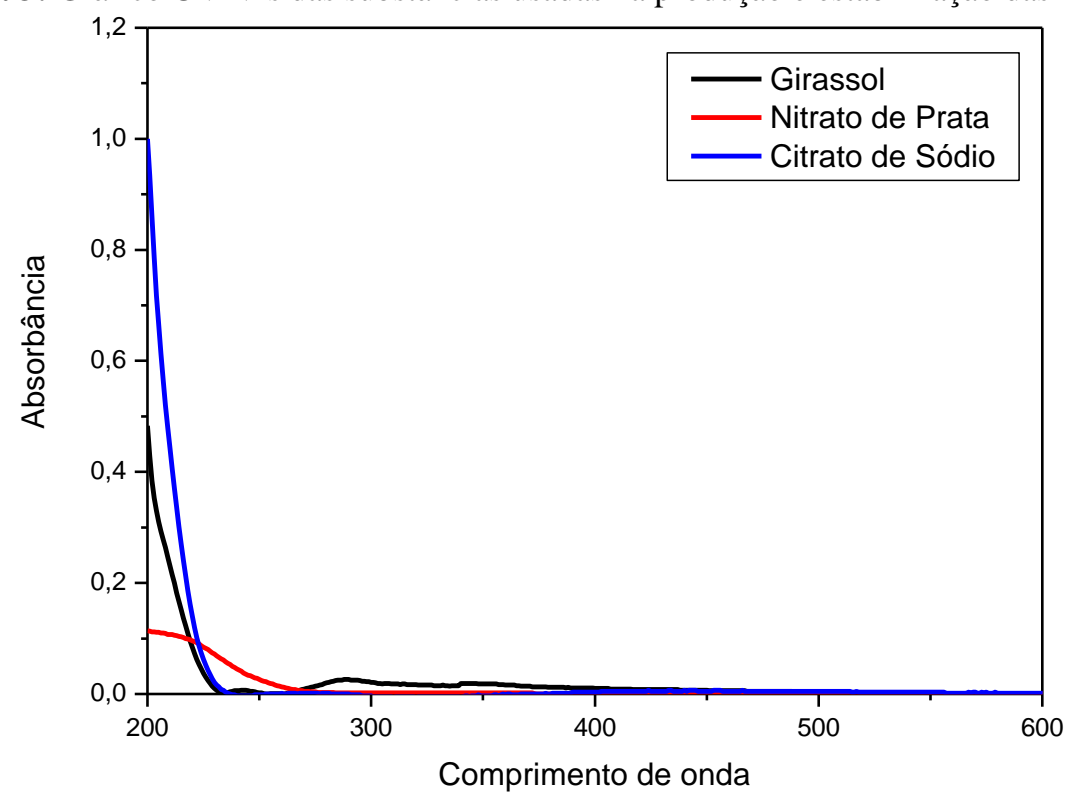

Fonte: Autores.

O segundo resultado obtido na caracterização foi os picos de UV-Vis derivados na nanopartícula na presença de citrato de sódio $1 \%$ e na estabilização com o extrato de sementes de girassol, representado pela Figura 3.1. Como observado as bandas de absorção de luz com comprimento de onda máximo próximo a $400 \mathrm{~nm}$, indicam que as nanopartículas de pratas (AgNPs) foram produzidas, ressaltado que quanto menor o comprimento de onda máximo, menor o tamanho das nanopartículas produzidas, o contrário também é verdadeiro.

Ao comparar as nanopartículas formadas na presença do citrato de sódio com ou sem a adição do extrato de girassol, percebemos que há uma redução do pico máximo de comprimento de onda, indo de 422 para 414 nm, o que por consequência nos leva a deduzir que ao ser adicionado o extrato de girassol a síntese produz nanopartículas menores, essa otimização das nanopartículas, facilita a toxicidade do material, já que quanto menor a nanopartícula mais facilidade a mesma tem de adentrar no nível celular e com isso ter atividade (Kim et al., 2012), assim como há a influência do tamanho da nanopartícula sobre a atividade antimicrobiana da substancia (Qasim et al., 2018). 
Figura 3.1: Gráfico UV-Vis das AgNPs produzidas na presença do citrato de sódio 1\% e estabilizada com o extrato de girassol.

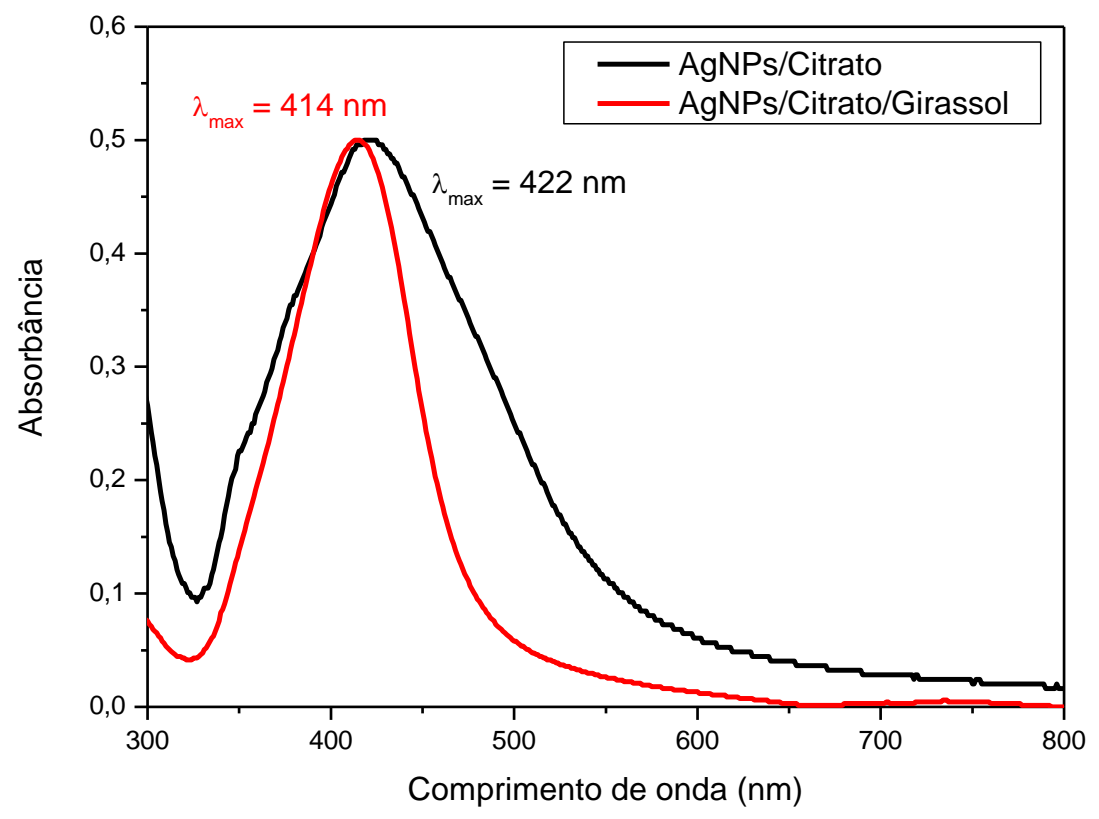

Fonte: Autores.

Na Figura 3.1 podemos observar o tamanho médio apresentado pelas nanopartículas ficou na ordem de $414 \mathrm{~nm}$ a 422 $\mathrm{nm}$ de comprimento da onda no maior volume da amostra, um pequeno volume das nanopartículas de prata apresentou uma faixa de tamanho que variou de $0,1 \mathrm{~nm}$ a $0,3 \mathrm{~nm}$ de absorbância.

\subsection{Avaliação da atividade antimicrobiana das nanopartículas}

A nanopartícula de prata é a nanoestrutura mais utilizada com o propósito de ação antimicrobiana dentre as nonopartículas projetadas devido a sua forte toxicidade para uma ampla gama de microrganismos, incluindo bactérias Grampositivas e Gram-negativas. (Liu et al., 2015; Tang; Zheng, 2018). Em adição a isso, a semente de girassol além de ser uma importante fonte de nutrientes, minerais e vitaminas, apresenta uma importante ação antimicrobiana, onde já foi demonstrando ação inibitória variada do extrato da semente de Helianthus annuus contra as espécies bacterianas e fungicas de Staphylococcus aureus, Bacillus subtilis, Vibrio cholera, Aspergillus fumigates e Candida albicans (Subashini; Rakshitha, 2012).

A medida do diâmetro dos halos de inibição (média das e repetições) revelou que a ação da nanopartícula de prata associada ao substrato de girassol foi de $16 \mathrm{~mm}$ para $S$. aureus (Figura 4) e de $12 \mathrm{~mm}$ e $10 \mathrm{~mm}$ para E. coli (Figura 5) e $P$. aeruginosa (Figura 6) respectivamente, demonstrando que a ação antibacteriana desta substância é maior contra bactérias gram-positivas (S. aureus) do que para gram-negativas (E. coli e P. aeruginosa). 
Figura 4: Teste de difusão em discos contendo nanopartícula de prata associada ao substrato de girassol (Ag + girassol) contra Staphylococcus aureus.

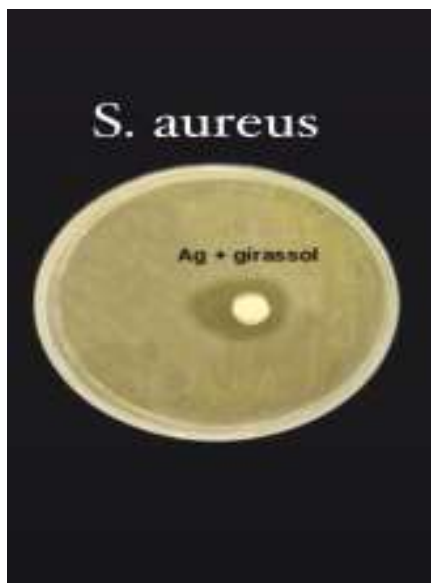

Fonte: Autores.

Figura 5: Teste de difusão em discos contendo nanopartícula de prata associada ao substrato de girassol (Ag + girassol) contra Escherichia coli.

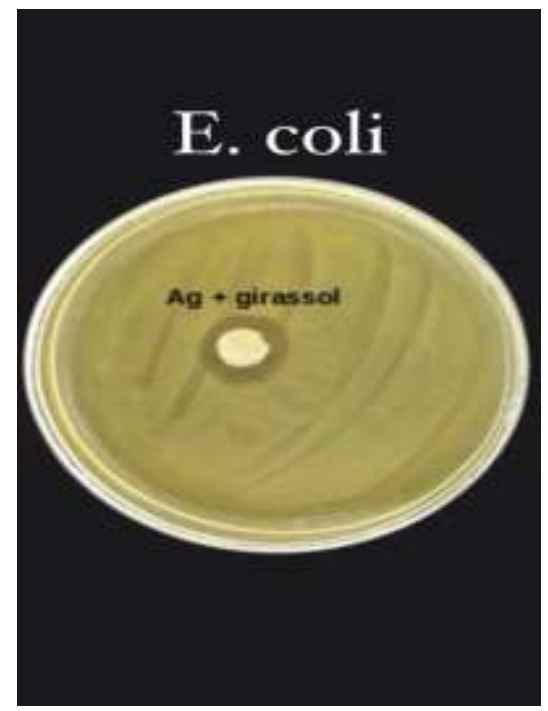

Fonte: Autores. 
Figura 6: Teste de difusão em discos contendo nanopartícula de prata associada ao substrato de girassol ( $\mathrm{Ag}+$ girassol) contra Pseudomonas aeruginosa.

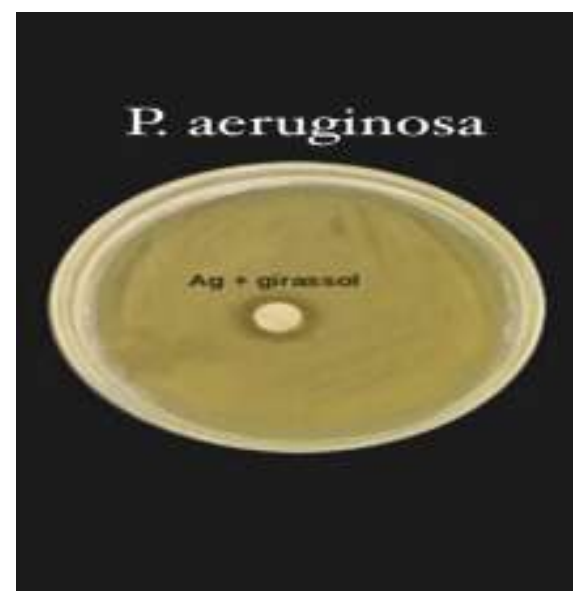

Fonte: Autores.

No ensaio de suscetibilidade por meio de disco difusão em meio sólido, observou-se a formação de halos de inibição do crescimento microbiano em todas as placas onde foram adicionados os discos contento a solução de AgNP-Ha, exceto nas amostras com Candida albicans (Figura 7), onde em nenhuma das amostras das triplicatas houve formação de halos inibitórios.

Figura 7: Teste de difusão em discos contendo nanopartícula de prata associada ao substrato de girassol $(\mathrm{Ag}+$ girassol) contra Candida albicans.

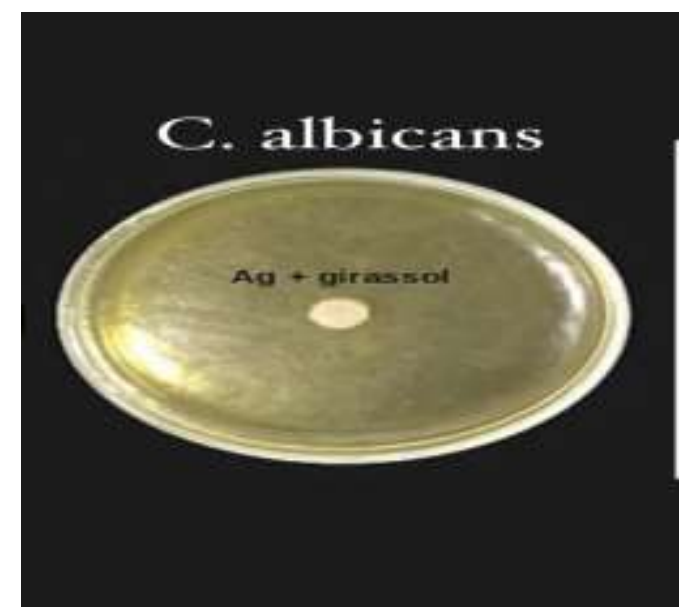

Fonte: Autores.

Em um estudo similar foi avaliada a atividade antimicrobiana de nanopartículas poliméricas à base de poli- $N$ isopropilacrilamida encapsulando nanopartículas de prata. Em concordância com o presente trabalho, o sistema de nanopartículas também apresentou efeitos contra E.coli e S. areus. Diferentemente da nanopartícula de prata estabilizada com girassol, foi relatado um maior efeito antimicrobiano contra $E$. coli do que $S$. aureus, fato que foi atribuído à diferença estrutural nas paredes das células bacterianas (Qasim et al., 2018). Semelhante à ação antibacteriana apresentada por nanopartículas de prata revestidas com glutationa, onde o efeito foi mais intenso em gram-negativas que em bactérias grampositivas (Taglietti et al., 2012).

As discordâncias entre os estudos podem ser atribuídas aos diferentes mecanismos de ação antimicrobiana que a nanopartícula de prata apresenta como: fixar-se na parede celular e na membrana, ocasionar danos de biomoléculas e estruturas 
intracelulares e promover estresse oxidativo induzido por íons de prata. Tais mecanismos podem agir de forma isolada como simultaneamente nas ações antibacterianas (Prabhu; Poulose, 2012).

Somado a isso, a composição do produto natural utilizado para estabilizar a nanopartícula influencia diretamente na atividade antimicrobiana. Devido à presença de compostos no extrato da semente de Helianthus annuus como flavonóides, alcalóides, saponinas e taninos que comprovadamente inativam as adesões microbianas, enzimas e proteínas de transporte do envelope celular (Tang et al., 2014).

\section{Conclusão}

Os extratos obtidos a partir da síntese verde do Girassol (Helianthus annuus) na redução do citrato de prata (AgNO3) se mostraram satisfatórios e promissores para a síntese de nanopartículas de prata.

$\mathrm{Na}$ análise de UV-Vis, foram observados uma dimensão em torno de 20 nanometros picos de absorbância que variaram de 414 a $422 \mathrm{~nm}(\lambda \max 422 \mathrm{~nm})$, que caracteriza a síntese de AgNPs que o extrato de Helianthus annuus a partir destes picos foram capazes de comprovar a estabilização.

As AgNPs sintetizadas apresentaram atividade bacteriostática e bactericida com presença de halo inibitório para $P$. aeruginosa $(10 \mathrm{~mm})$, S. aureus $(16 \mathrm{~mm})$, E. coli $(12 \mathrm{~mm})$ e na espécie fúgica Candida albicans não foi observado formação de halo inibitório. São necessários ainda a realização de mais testes de ação antibacteriana e antifúngica para determinar se as NPs obtidas pela síntese verde com extrato de Helianthus annuus apresentam a mesma eficácia que as obtidas através de outras plantas e/ou métodos. Sugerindo assim mais estudos para realizar a otimização dos parâmetros de síntese.

\section{Referências}

Amkhande, P. G., et al, "Metal nanoparticles synthesis: An overview on methods of preparation, advantages and disadvantages, and applications", Journal of Drug Delivery Science and Technology, 53, 101174

Al-Shabib, N. A. et al. Bio-inspired facile fabrication of silver nanoparticles from in vitro grown shoots of Tamarix nilotica: explication of its potential in impeding growth and biofilms of Listeria monocytogenes and assessment of wound healing ability. RSC Advances, 10, 30139-30149

Atiyeh, B. S, Costagliola, M., \& Hayek, S. Effect of silver on burn wound infection control and healing: review of the literature. Burns 33 (2): 139-48 Dibo SA

Bauer, A. W., et al. (1966) Antibiotic susceptibility testing by a standardized single disk method. American Journal of Clinical Pathology, $45,493-496$.

Brinch, A., et al. EU regulation of nanobiocides: challenges in implementing the biocidal product regulation (BPR). Nanomaterials, 6, 33

Chaudhuri, R. G., \& Paria, S. Core/shell nanoparticles: classes, properties, synthesis mechanisms, characterization, and applications. Chemical Reviews, 112, $2373-433$

CLSI. Clinical and Laboratory Standards Institude. Performance standards for antimicrobial disk susceptibility tests, approved standard-tenth edition M02A10. 29.

Dasa, P., Ghosalb, K., Jana, N. K., et al. "Green synthesis and characterization of silver nanoparticles using belladonna mother tincture and its efficacy as a potential antibacterial and anti-inflammatory agent”, Material Chemistry and Physics 228, 310-317

Deshmukha, S. P. et al. "Silver nanoparticles as an effective disinfectant: A review”, Materials Science \& Engineering C 97, 954-965

Georgiev, P. et al. Implementing atomic force microscopy (AFM) for studying kinetics of gold nanoparticle's growth. Colloids and Surfaces A: Physicochemical and Engineering Aspects, 434, 154-163

Guo, S., Ge, Y., \& Jom, K. A review of phytochemistry, metabolite changes, and medicinal uses of the common sunflower seed and sprouts (Helianthus annuus L.). Chemistry Central Journal. 11, 1-10

Hong, H. R., et al. Facile fabrication of multifunctional fabrics: use of copper and silver nanoparticles for antibacterial, superhydrophobic, conductive fabrics. RSC advances, 8, 41782-41794

Ibrahim, T. A., Ajongbolo, K. F., \& Aladekoyi, G. Phytochemical screening and antimicrobial activity of crude extracts of Basella albaand Helianthus annuuson selected food pathogens. Journal of Microbiology and Biotechnology, 3(2): 27-31 
Junior, M. A. Melo, Santos, L. S. S., Gonçalves, M. C., \& Nogueira, A. F. Preparação de nanopartículas de prata e ouro: um método simples para a introdução na nanociência em laboratório de ensino. Quim. Nova, 35, 1872-1878Khan, I., Saeed, K., Khan, I. Nanoparticles: Properties, applications and toxicities. Arabian Journal of Chemistry

Kim, T. H., et al. Size-dependent cellular toxicity of silver nanoparticles. Journal of biomedical materials research Part A, 100, 1033-1043.

Khodashenas, B., \& Ghorbani, H. R. Synthesis of silver nanoparticles with different shapes. Arabian Journal of Chemistry, p. 1-16

Lemire, J. A., Harrison, J. J., \& Turner, R. J. Antimicrobial activity of metals: mechanisms, molecular targets and applications. Nature Reviews Microbiology, $11,371-384$

Liu, Z. et al. Catalytic and antibacterial activities of green-synthesized silver nanoparticles on electrospun polystyrene nanofiber membranes using tea polyphenols. Composites Part B: Engineering, 79, 217-223

Lucky, S. S., Soo, K. C., \& Zhang, Y. Nanoparticles in photodynamic therapy. Chemical reviews, 115, 1990-2042

Mishra, A. et al. Reusable Green Synthesized Biomimetic Magnetic Nanoparticles for Glucose and H2O2 Detection. BioNanoScience, 6, 93-102

Mello, S. O. N. et al. In vitro mutagenicity assay (Ames test) and phytochemical characterization of seeds oil of Helianthus/ annuus Linn (sunflower). Toxicology Reports, 3: 733-739, 2016. [CrossRef]

Nath, D., \& Banerjee, P. Green nanotechnology - a new hope for medical biology. Environmental Toxicology and Pharmacology, 36, 997-1014

Park, Keunju, Seo, Dongseok, Lee, Jongkook. Conductivity of silver paste prepared from nanoparticles. Colloids and Surfaces A: Physicochemical and Engineering Aspects, 313, 351-354,

Pinto, C. P., et al. et al. Antimicrobial activity of Lippia species from the Brazilian semiarid region tradionally used as antiseptic and anti-infective agents. Evidence-Based Complementary and Alternative Medicine, 2013, 1-5

Prabhu, S., \& Poulose, E. K. Silver nanoparticles: mechanism of antimicrobial action, synthesis, medical applications, and toxicity effects. International nano letters, 2, 1-10

Qasim, M. et al. Antimicrobial activity of silver nanoparticles encapsulated in poly-N-isopropylacrylamide-based polymeric nanoparticles. International journal of nanomedicine 13, 235, 2018.

Qiao, Z. et al. Extraction, Radical Scavenging Activities, and Chemical Composition Identification of Flavonoids from Sunflower (Helianthus annuus L.) Receptacles. Molecules, 26, 403

Ravindra, S. et al. Fabrication of antibacterial cotton fibres loaded with silver nanoparticles via "Green Approach". Colloids and Surfaces A: Physicochemical and Engineering Aspects, 367, 31-40

Ratner, B., et al. Biomaterials Science: An Introduction to Materials in Medicine. (3a ed.), Academic Press.

Souza, T. A. J., Souza, L. R. R., \& Franchi, L. P., "Silver nanoparticles: An integrated view of green synthesis methods, transformation in the environment, and toxicity", Ecotoxicology and Environmental Safety, 171, 691-700

Singh, J., Dutta, T., Kim, K. H., Rawat, M., Samddar, P., \& Kumar, P. 'Green' synthesis of metals and their oxide nanoparticles: applications for environmental remediation. Journal of Nanobiotechnology, 16, 1-24

Subashini, R., \& Rakshitha, S. U. Phytochemical screening, antimicrobial activity and in vitro antioxidant investigation of methanolic extract of seeds from Helianthus annuus L. Chemical science review and letters, 1, 30-34

Swietnicki, W. Recent advances in antibacterial drug development. International Journal of Recent Scientific Research, 9, 26501-26505

Taglietti, A. et al. Antibacterial activity of glutathione-coated silver nanoparticles against gram positive and gram negative bacteria. Langmuir, 28, 8140-8148

Tang, D. et al. A review of phytochemistry, metabolite changes, and medicinal uses of the common food mung bean and its sprouts (Vigna radiata). Chemistry Central Journal, 8, 1-9

Tang, S., \& Zheng, J. Antibacterial activity of silver nanoparticles: structural effects. Advanced healthcare materials, 7, 1701503

Thierry, B. Drug nanocarriers and functional nanoparticles: applications in cancer therapy. Current drug delivery. 6, 391-403 